\title{
Pluralité des acteurs et pratiques inclusives: les paradoxes de la collaboration
}

Résumé: Après avoir rappelé que la nouvelle donne de la loi de 2005 rend inéluctable le choc de deux cultures en ce qu'elle modifie la grammaire professionnelle des acteurs, cet article décrit leur nouveau cadre d'intervention comme un continuum d'action éducative structuré autour du repère commun de la scolarisation et délivré des frontières entre les territoires institutionnels. II examine ensuite, en s'appuyant sur l'analyse de situations de handicap, la nouvelle articulation des concepts de référence à travers trois oppositions structurantes: différence et singularité, déficience et fonctionnement, trouble et besoins.

Mots-clés: Accessibilité - Besoin éducatifs particuliers - Continuum - Coopération - Diversité - Établissement spécialisé - Handicap - Inclusion - Intégration - Médiation - Scolarisation en milieu ordinaire.

\section{The Paradoxes of Cooperation Practices in Inclusive Settings}

Summary: First, this paper describes how the new deal of Law 2005-102 of 2005 makes inevitable the clash of two cultures, as it modifies the professional paradigm of stakeholders, then, it analyses the new settings where educational interventions take place as a continuum of action structured by the common standpoint of mainstreaming and cleared out by frontiers between institutional territories. At last, with examples of disabled people situations, it focuses on the new system of referential concepts, through three significant oppositions: difference and peculiarity, impairment and functioning, disorder and needs.

Keywords: Accessibility - Continuum - Cooperation - Disability - Diversity - Inclusion - Integration Mainstreaming - Mediation - Special educational needs - Special schools

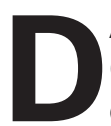

ANS un ouvrage intitulé L'école face aux handicaps, Éric Plaisance et Michel Chauvière (2000) écrivaient à propos des deux secteurs éducatifs, celui des établissements spécialisés et celui de l'École: "Depuis que ces deux secteurs éducatifs coexistent, [...] il semble qu'ils œuvrent dos à dos. " Dans le récent hors série de La nouvelle revue de l'adaptation et de la scolarisation, intitulé "L'éducation inclusive en France et dans le monde ", Sylvie Canat (2009, p. 149), estime que la loi du 11 février 2005 " demande à ces deux secteurs de se regarder en face ". La rencontre et l'articulation sur de nouvelles bases de deux cultures professionnelles différentes, adossées à leurs modèles théoriques propres et à des référentiels de pensée distincts, voire divergents, imposent à la fois aux 
professionnels, aux formateurs et aux chercheurs des remaniements en profondeur de leurs pratiques, de leurs conceptions et de leurs méthodes de travail. II est donc essentiel de définir et de caractériser le new deal de la loi de 2005, afin de montrer comment il rend inéluctable le choc de deux cultures, dont la cohabitation dans le champ éducatif n'avait donné lieu jusque-là qu'à des conflits marginaux, des escarmouches interinstitutionnelles qui ne remettaient aucunement en question le fonctionnement général du système dans son ensemble.

Selon Michel Chauvière et Éric Plaisance, c'est entre 1960 et 1970 que se développent les premières critiques de l'éducation ségrégative et que la légitimité de la séparation entre éducation scolaire et éducation spéciale commence à être remise en question. Ainsi verra-t-on la naissance des Gapp (Groupes d'aide psychopédagogique) en 1970. C'est dans le cadre de ce courant de pensée de désinstitutionnalisation que l'Italie, dès 1971, par la loi $n^{\circ} 118$, adopte la première mesure officielle de placement des élèves handicapés en milieu ordinaire, sans toutefois statuer sur le maintien ou la fermeture des écoles spécialisées, ce qui laissait, comme l'écrit Simona D’Alessio (2009), une marge de manœuvre aux partisans du "dualisme éducatif » (Benoit 2003). La loi française du 30 juin 1975 encouragera elle aussi clairement l'accueil en milieu scolaire ordinaire des enfants et adolescents handicapés, chaque fois qu'il est possible " malgré leur handicap " (article 5-1), mais en maintenant, "à défaut " (article 4) la possibilité d'une "éducation spéciale ". La structure du système éducatif n'était donc pas bouleversée puisque subsistaient deux voies distinctes d'éducation, même si l'on préconisait les échanges entre l'une et l'autre. II en va différemment de la loi du 11 février 2005, qui, comme l'avait fait presque 30 ans plus tôt la loi italienne n 517 de 1977, veut intégrer l'ensemble des structures et services de l'ancienne éducation spéciale dans un système unifié d'éducation fondé sur la référence à la scolarisation. Bien qu'il ne s'agisse en aucun cas dans l'esprit de la loi de 2005 d'engager, comme en 1977 en Italie, le démantèlement de la filière spécialisée, le changement est profond, car il implique de "faire culture commune ", comme le pensent Charles Gardou et Yves Jeanne (2008). L'enjeu est donc lié à la question stratégique des conditions l'articulation entre les établissements scolaires et les établissements médico-éducatifs que la loi de 1975 s'était abstenue de régler (Chauvière et Plaisance, 2008).

Qu'il s'agisse de faire cause commune ou de faire culture commune, l'expression met bien en évidence que les protagonistes (c'est-à-dire les acteurs de l'éducation spécialisée et ceux de l'école ordinaire) se donnent un but commun, auquel ils concourent avec leurs savoirs et leurs compétences respectifs. Cette formulation en apparence anodine renvoie à la question centrale du sens que les différents professionnels donnent à leur mission et met en évidence que le point de blocage réside peut-être tout autant dans l'identification de l'objectif visé que dans les moyens mis en œuvre pour l'atteindre. Le paradoxe actuel du professionnel spécialisé du secteur médico-éducatif est qu'il tient historiquement son identité professionnelle du modèle de l'expert, celui qui résout et prescrit à la place de, qui agit par délégation dans un contexte fortement empreint d'une culture bio-médicale, et qu'il se trouve maintenant confronté à des pratiques de l'avec, adossées à des logiques sociales de collaboration, d'accompagnement et de co-intervention (Vallerie, 2010). II en résulte qu'il ne lui est plus possible de penser son domaine 
de compétence comme un territoire qui s'arrêterait là où commence celui de ses collègues du milieu ordinaire, mais qu'il lui faut intégrer l'idée que ces deux domaines se recouvrent et se superposent. Ses fonctions ne relèvent plus du registre de la substitution, mais de celui de la combinaison et de l'articulation des compétences dans la dynamique d'un réseau de ressources: il s'agit donc pour lui de passer d'une grammaire professionnelle de type paradigmatique, dans laquelle les déclinaisons de chaque type d'interventions professionnelles se déroulaient parallèlement, à une grammaire de type syntagmatique, où les rôles interagissent en permanence dans un environnement continu, structuré par ses objectifs et non plus par ses frontières.

\section{UN NOUVEAU CADRE PROFESSIONNEL: LE CONTINUUM TERRITORIAL D'ACTION ÉDUCATIVE}

\section{Une voie scolaire ordinaire de référence}

C'est parce qu'elle a mis en place un système unifié d'éducation fondé sur la référence à la scolarisation que la loi instaure un continuum territorial d'action éducative. Pour caractériser cette mutation de structure, on peut s'appuyer dans un premier temps sur la typologie élaborée par l'Agence européenne pour positionner les politiques éducatives des pays de I'Union au regard de I'objectif d'éducation inclusive (Champollion, 2007; Benoit, 2008b). II existe à ses yeux trois grands types de politiques:

- "Two tracks approach »: cette option est celle des pays où l'on pratique deux voies de scolarisation séparées, l'une ordinaire, l'autre spécialisée, pratiquement exclusives l'une de l'autre. En Belgique, par exemple, 97 \% des jeunes handicapés sont systématiquement scolarisés dans des écoles spéciales.

- "Multi tracks approach »: ce choix est celui des pays qui ont une approche multiple et pragmatique de l'éducation des jeunes handicapés et qui développent aussi bien l'intégration scolaire que l'éducation spéciale dans des établissements séparés. L'Allemagne est un exemple de ces pays.

- "One track approach »: I'Italie adopté cette politique qui consiste à scolariser systématiquement les enfants et adolescents handicapés dans les classes ordinaires (mainstreaming).

La France qui, après la loi de 1975 et le développement à partir des années 19821983 de l'intégration scolaire, se situait dans le type "Multi tracks approach ", s'est rapprochée du type "One track approach » depuis I'instauration par la loi du 11 février 2005 du droit pour chaque famille d'inscrire son enfant handicapé dans l'établissement scolaire "le plus proche de son domicile ${ }^{1}$ ». Non pas à la manière radicale de I'Italie, mais en substituant au principe de filiarisation des jeunes handicapés, fondé sur le processus d'orientation, celui de "parcours de formation 2 ", adossé aux deux notions de projet et d'accompagnement, et obligatoirement référé à la voie ordinaire d'éducation, aussi bien du point de vue géographique que programmatique. Ainsi les lieux traditionnels d'éducation spécialisés ne sont-ils pas rayés de la carte, mais

1. Loi 2005-102 du 11 février 2005, titre IV, article 19, III.

2. Décret $n^{\circ}$ 2005-1 752 du 30 décembre 2005 « relatif au parcours de formation des élèves présentant un handicap $"$. 
deviennent en principe des modalités éducatives à référence externe, c'est-à-dire que leur action ne prend son sens qu'au regard de leur contribution préparatoire et complémentaire aux apprentissages prévus dans le cursus ordinaire. Ces structures n'ont plus vocation à se substituer à l'établissement ordinaire "de référence ${ }^{3}$ ", dans le cadre d'une filière spécifiquement dédiée à la mise en œuvre au long cours de diverses prestations réparatrices, médicale, éducative, scolaire et sociale, mais à organiser un accueil temporaire, n'excluant aucunement, dans son principe, la scolarisation en milieu ordinaire.

II ne s'agit donc plus désormais, comme dans le cadre antérieur du dualisme éducatif, fondé sur la coexistence d'établissements d'éducation spéciale et de structures scolaires de droit commun, de passer des ponts entre les deux filières ordinaire et spécialisée (Benoit, 2008b, p. 100). Le processus d'intégration scolaire, qui était réservé à des élèves supposés capables de satisfaire aux exigences scolaires, se voit remplacé par un processus inclusif destiné à répondre à la diversité des besoins individuels de tous. La question n'est plus de mettre le jeune handicapé à l'épreuve de la norme scolaire, mais d'interroger le système éducatif sur sa capacité à prendre en compte la diversité des besoins particuliers de tous les élèves, notamment lorsque ceux-ci sont liés à une déficience, un trouble ou une maladie. On s'inscrit alors dans une perspective renversée, où ce n'est plus seulement à l'élève de s'adapter à une norme scolaire intangible, mais aussi à l'école de s'adapter à la diversité des élèves en reconnaissant la singularité de chacun. C'est là une nouvelle logique qui engage à rechercher les moyens de la mise en accessibilité des cursus de droit commun, c'est-à-dire de leur accessibilisation.

\section{Évolution des référentiels de l'action professionnelle}

Les conséquences en termes de référentiels d'action professionnelle pour les acteurs des deux anciennes filières sont considérables. II s'agit d'abord, pour les uns et les autres, d'abandonner les repères antérieurs en termes de filières et d'adopter la référence commune du parcours de formation, dans l'esprit du modèle social de la CIF (Classification internationale du fonctionnement, du handicap et de la santé), selon lequel l'objectif de participation sociale de l'enfant et de l'adolescent se réalise par l'exercice plein et entier dans les structures scolaires de droit commun de son statut d'élève et débouche sur une insertion sociale et professionnelle en milieu ordinaire de vie. Alors que la scolarisation en milieu ordinaire ici et maintenant constitue la visée de l'intégration, elle ne représente pas au titre de l'inclusion "une fin en soi " (Armstrong F., Armstrong D., Barton L., 2000) et ne prend tout son sens qu'à partir du moment où elle embrasse à la fois toutes les phases du parcours de vie et tous les systèmes sociaux et culturels qui pourront avoir sur lui un impact direct ou indirect. II en résulte que la question principale à se poser dans cette perspective n'est plus celle de l'orientation ${ }^{4}$ de l'enfant vers la structure adaptée à ses besoins, mais

3. Loi 2005-102 (ibidem).

4. La fonction d'orientation, qui était principalement dans les faits celle des commissions mises en place par la loi de 1975 et supprimées par la loi de 2005, avait été mise en cause dès 2002 (circulaire $n^{\circ} 2002-112$ du 30.04.2002): "Nous rappellerons [...] (que) la vocation première des commissions est de proposer un projet d'intégration en milieu ordinaire avant d'envisager une scolarisation en milieu spécialisé. " 
celle de son accompagnement dans un cursus scolaire appelé en permanence à se rendre accessible et capable de produire les réponses adaptatives nécessaires. Ce sont donc des dispositifs plutôt que des structures, des services, plutôt que des établissements, qui constituent les moyens adéquats de mise en place d'une telle politique.

Il est tout à fait significatif à cet égard que l'outil régulateur du parcours de formation de l'élève handicapé se nomme projet personnalisé de scolarisation et qu'il regroupe l'ensemble des mesures éducatives rééducatives, thérapeutiques, sociales dont bénéficie le jeune handicapé. En même temps que les deux filières se fondent en une seule, celle de la scolarisation, disparaît la dichotomie entre ce que l'on appelait auparavant le PIIS (Projet individualisé d'intégration scolaire), à la charge des enseignants de la voie ordinaire, et le projet éducatif et thérapeutique individualisé, que devaient réaliser les professionnels du secteur médico-éducatif. La création du PIA (Projet individualisé d'accompagnement) par le décret et l'arrêté du 2 avril 2009 ne reproduit d'ailleurs en aucune façon la configuration antérieure puisque le PIA est défini comme la mise en œuvre du PPS par un établissement ou service spécialisé. Le champ professionnel des acteurs concernés par l'éducation des élèves handicapés se trouve ainsi unifié et structuré par un nouveau repère, au sens géométrique du terme, qui le réorganise de manière hiérarchisé autour d'un but commun: la scolarisation. On voit ainsi se dessiner, au-delà des ruptures ségrégatives, la réalité en construction d'un continuum scolaire, au niveau spatial (la classe ordinaire) et temporel (le cursus scolaire), dans lequel doivent s'inscrire et prendre sens les interventions spécialisées des personnels du secteur médico-social.

Ceux-ci sont désormais invités à adopter, au regard de leurs cadres traditionnels de fonctionnement, structurés autour de l'enfant objet de soins, à rééduquer, à protéger, une visée à repérage externe, c'est-à-dire à se décentrer de leurs pratiques, comme c'est par exemple le cas pour l'Unité d'enseignement (UE). Ce dispositif, que chaque établissement ou service spécialisé a vocation à créer, a pour but d'assurer la continuité du parcours de formation, c'est-à-dire de préparer et de compléter l'enseignement reçu en milieu scolaire ordinaire, pour des " enfants ou adolescents qui ne peuvent effectuer leur scolarité à temps plein dans une école ou un établissement scolaire ${ }^{5} "$. Une telle formulation présente en creux la scolarité partagée entre milieu ordinaire et milieu spécialisé comme une solution naturelle. Symboliquement, le législateur a d'ailleurs tenu à marquer le caractère " particulier et indissoluble ${ }^{6}$ " du lien qui doit être constamment maintenu entre l'unité d'enseignement et le milieu scolaire ordinaire en disposant, d'une part, que, pour l'élève handicapé, son "établissement scolaire de référence le reste [...] sous la forme d'une inscription inactive [...] dans le cas où le PPS rend nécessaire son inscription dans un autre établissement ${ }^{7}$ ", et, d'autre part, qu'en cas de scolarisation partielle au sein d'un établissement sanitaire ou médico-social, il ne peut être administrativement inscrit (inscription active) que dans un établissement scolaire ordinaire proche de cet établissement spécialisé.

\footnotetext{
5. Ibidem, article 14

6. Circulaire 2006-126 du 17 août 2006, portant « Mise en œuvre et suivi du projet personnalisé de scolarisation ». 7. Ibidem.
} 
La convention de coopération qui sera alors établie entre les deux établissements privilégiera l'accueil en classe ordinaire, en évitant toute " fréquentation occasionnelle ou réduite à quelques heures par semaine [...] (qui) serait contraire à l'idée même du PPS ${ }^{8} "$.

Le fait que le champ de compétence de l'enseignant référent, dont le rôle est de veiller à la continuité et à la cohérence du parcours de formation des élèves handicapés, inclue les établissements spécialisés témoigne de la volonté d'éviter toute solution de continuité entre le traitement des élèves accueillis dans les structures médicoéducatives et les autres: réunie et animée par l'enseignant référent, l'équipe de suivi de la scolarisation, dont la mission est d'assurer le suivi du PPS et de veiller au bon déroulement du parcours scolaire, a droit de cité au sein des établissements spécialisés, où les traditionnelles réunions de synthèse, le plus souvent internes, ne sont plus le seul cadre de référence.

Dans le continuum scolaire, qui est aussi celui des collaborations éducatives, les frontières anciennes entre les territoires institutionnels, qui fonctionnaient comme des sources de reconnaissance identitaires, sont destinées à s'effacer progressivement. Ces frontières, ce sont celles qui délimitaient la classe spécialisée, lieu de référence et véritable port d'attache pour l'élève handicapé ou en difficulté, celles qui le séparaient du milieu ordinaire, où il s'engageait dans un voyage intégratif, et d'où, en cas de problème, il pouvait à tout moment être rejeté. Ce sont celles qui dissociaient la voie ordinaire de scolarisation de la filière spécialisée et généraient la précarité du statut d'élève en intégration scolaire; ce sont celles qui passaient entre une culture fondée sur l'enfant, la santé et le soin et une culture fondée sur l'élève, la vie sociale et les apprentissages.

Les premiers " dispositifs collectifs d'intégration " que sont les UPI 9 (Unités pédagogiques d'intégration), récemment devenues Ulis ${ }^{10}$ (Unités localisées pour l'inclusion scolaire), spécifient par exemple que les élèves scolarisés au titre de I'Ulis sont des élèves à part entière de l'établissement et que leur inscription se fait dans la division ${ }^{11}$ correspondant à leur Projet personnalisé de scolarisation (PPS). Ainsi la scolarisation de ces élèves est-elle clairement référée aujourd'hui à la classe ordinaire, lieu de diversité, et non plus à un espace spécialisé parallèle.

\section{UNE NOUVELLE ARTICULATION DES CONCEPTS DE RÉFÉRENCE}

Les professionnels sont également confrontés à une évolution radicale des conceptions: on est passé de l'idée qu'un enfant handicapé est éducable, à l'idée que c'est un enfant qui, comme tous les autres, a droit à une scolarisation en milieu scolaire ordinaire. Cela implique concrètement que I'on renonce à classer l'enfant handicapé dans une catégorie diagnostique, qui déterminera son affectation dans la structure spéciale correspondante, avant de le scolariser. Cela implique, de manière sous-jacente, une profonde mutation en termes de modèles de pensée de référence,

\footnotetext{
8. Ibidem

9. Circulaire 2001-035 du 21 février 2001.

10. Circulaire 2010-088 du 18 juin 2010.

11. Il faut entendre par là le niveau de classe $\left(6^{e}, 5^{e} \ldots\right)$, ce qui implique que I'Ulis ne constitue pas une division.
} 
que I'on peut faire apparaître à travers trois couples de notions antagoniques qui correspondent à des oppositions structurantes:

- différence et singularité,

- déficience et fonctionnement,

- trouble et besoin.

\section{Différence ou singularité?}

La première opposition est celle qui confronte le modèle de pensée fondé sur la différence au modèle de pensée fondé sur la singularité. C'est parce que l'on a considéré très longtemps les enfants et adolescents handicapés comme radicalement différents, absolument différents, que l'on avait organisé pour eux une éducation séparée dans des lieux de soins, dans des lieux de réparation, où l'éducation, et a fortiori la scolarisation, venaient en second lieu, quand l'enfant allait mieux et que l'on décidait que son état de santé les rendait possibles.

On dispose aujourd'hui de fortes raisons pour penser les singularités individuelles en termes de continuum de diversité plutôt qu'en termes de différences et de ruptures. C'est parce que l'on est devenu capable de concevoir et de prendre en compte, non plus la différence, mais la singularité de chaque enfant, qu'on a pu envisager la scolarisation en milieu ordinaire. On s'est rendu compte que l'appartenance à une même catégorie diagnostique ne garantit nullement que les enfants présentent des besoins éducatifs identiques. En revanche, la séparation du groupe de pairs limite les interactions et les fondements médico-sociaux de l'éducation spéciale conduisent à privilégier des objectifs de socialisation, dans le cadre d'actions thérapeutiques, les apprentissages n'intervenant que secondairement. II en résulte un effet de médicalisation et de filiarisation, peu propice, à terme, à l'acquisition des compétences scolaires et de la formation professionnelle indispensables à l'intégration sociale. Ce renversement de perspective qui voit la classe ordinaire, lieu de diversité, devenir la référence, en lieu et place de la classe spéciale, adossée à la catégorie, renvoie aussi bien les enseignants ordinaires que les personnels spécialisés à un nécessaire remaniement de leurs conceptions. Les premiers, encore imprégnés des modèles pédagogiques traditionnels qui postulent la conformité à un idéal d'élève performant intellectuellement, voire en bonne santé, ressentent la scolarisation des jeunes handicapés en milieu ordinaire comme une injonction irréaliste, impossible à appliquer concrètement (Chauvière et Plaisance 2008, p. 38). Les seconds, en retour, véhiculent parfois une vision ambivalente des contraintes liées à I' " obligation scolaire "; leur perception de la scolarisation prend la forme soit d'une action complémentaire aux soins, accessoire mais bénéfique, soit d'un danger de mise en souffrance pour des enfants déjà suffisamment fragilisés par leur trouble. L'École apparaît alors à ces professionnels spécialisés comme, "au choix, la cerise sur le gâteau, la mouche du coche ou le loup dans la bergerie " (Horvais, 2008).

C'est bien pourtant la reconnaissance du droit à la singularité (Benoit, 2008b), en dehors de toute stigmatisation, qu'affirme la loi du 11 février 2005, par le droit pour tout élève d'être inscrit dans l'école de son quartier - celle qu'il aurait fréquenté s'il n'avait pas été handicapé. Et l'on voit bien l'intérêt de ce droit, non seulement pour les enfants en situation de handicap, mais aussi pour tous les 
élèves. II y a là un enjeu de culture professionnelle enseignante qui comporte une autre implication importante: le dépassement de la notion de l'hétérogénéité des élèves par celle de l'hétérogénéité des compétences de chaque élève. La reconnaissance de l'hétérogénéité des élèves - lieu commun de la pédagogie depuis plus de vingt-cinq ans - renvoie en effet essentiellement au niveau scolaire et I'on a vu cette question du niveau revenir de façon récurrente au cœur des problèmes qui tendent à verrouiller les parcours de scolarisation des jeunes en situation de difficulté scolaire: "il/elle n'a pas pu continuer, il/elle n'avait pas le niveau ". Au-delà de la prise en compte de l'hétérogénéité des élèves, il y a celle de l'hétérogénéité des compétences. Cela signifie que pour chaque enfant, chaque élève, il s'agit de définir un profil de compétences qui peut être plus ou moins hétérogène et que c'est cette hétérogénéité-là qu'il convient de travailler dans le champ de sa singularité. II s'agit donc d'aller au-delà de l'hétérogénéité des élèves, qui renvoie d'une certaine façon à la différence, pour reconnaître l'hétérogénéité des compétences, qui, elle, renvoie à la singularité et à la diversité. Lorsque l'on examine, par exemple, des profils individuels d'enfants avec troubles envahissant du développement et que l'on découvre une observation comme celle-ci : "une excellente mémoire, il veut réussir très vite, il a du mal à revenir sur ses erreurs " on est frappé par le fait que ces caractéristiques, ici celles d'un jeune autiste, se rencontrent bien souvent chez des enfants ordinaires. L'existence de ces besoins communs à l'ensemble des enfants est ce qui fonde la possibilité d'un parcours de scolarisation de tous dans la voie ordinaire. II reste que la mise en œuvre de réponses scolaires adaptées à la singularité des besoins particuliers de chacun d'entre eux en est la condition indispensable. Et que les pratiques enseignantes sont loin du compte. En revanche, dans ce domaine de l'individualisation de la démarche éducative, de nombreux auteurs reconnaissent à l'éducation spécialisée une réelle avance sur l'éducation scolaire. II reste à mutualiser cette compétence et à la mettre au service des mêmes objectifs.

\section{Déficience ou fonctionnement?}

Le deuxième couple de notions oppose d'un côté le référentiel de pensée fondé sur le manque, sur la déficience, sur le défaut, sur la défaillance, et de l'autre le référentiel fondé sur le fonctionnement. La question du fonctionnement de l'enfant, la question de savoir comment fonctionnent les enfants en situation de handicap, que celle-ci soit liée à une déficience sensorielle, un trouble moteur ou un trouble des fonctions cognitives, est aujourd'hui au centre des préoccupations des pédagogues et de tous ceux qui concourent à l'éducation. Outre que la nouvelle Classification internationale du fonctionnement, du handicap et de la santé (CIF) adoptée par l'OMS en 2001 met le mot fonctionnement en pleine lumière, puisque la CIF se substitue à la $\mathrm{ClH}$ (Classification internationale du handicap), et que la $\mathbf{F}$ de fonctionnement se substitue à la $\mathbf{H}$ de handicap, il y a là une importante mutation de point de vue: on pourrait dire que l'on enterre la $\mathrm{H}$ du handicap. C'est le modèle de pensée de référence qui est en jeu: le modèle du fonctionnement est en effet très différent de celui du manque, qui a longtemps structuré une approche principalement médicale du handicap. On a pu parler d'approche défectologique (incapacités, désavantages, 
déficience). II y avait, dans la plupart des pays de l'ex-bloc de l'Est des facultés de défectologie.

On sait aujourd'hui que les déficits initiaux qui peuvent caractériser des personnes donnent lieu pour chacune d'entre elles à des destins variés, pour aboutir à un éventail très large de situations individuelles. C'est que le développement psychique et psychologique procède d'une structure globale et non pas d'éléments qui seraient juxtaposés les uns aux autres. II en résulte que l'altération d'une fonction peut être compensée par un nouveau fonctionnement réorganisé autour d'autres fonctions. Dans ces conditions, les particularités individuelles que l'on a tendance à isoler en terme de déficience se trouvent intégrées dans ce développement, c'est-à-dire qu'elles peuvent être plus ou moins compensées par un fonctionnement psychique réorganisé autour d'autres potentialités perceptives ou cognitives (Genevois, 2003). Les caractéristiques personnelles, par exemple un déficit sensoriel, moteur ou cognitif, ne structurent pas directement le fonctionnement de tel ou tel individu; leur impact sur son développement psychologique et, en bout de course, sur son fonctionnement, dépend en grande partie de la qualité de son environnement, ainsi que des médiations parentales, éducatives et pédagogiques dont il aura pu bénéficier (climat affectif, aides aux apprentissages et à la scolarisation). On voit bien l'intérêt qu'une telle approche peut avoir en termes d'action pédagogique. Ce qui nous importe, c'est le fonctionnement psychologique et cognitif de l'élève, plus que la référence à une liste de défaillances - défaillance de communication, par exemple - et dès lors que l'on cesse de penser en termes de manque et qu'on se met à penser en termes de fonctionnement, on se rend compte que le problème de l'enfant peut être formulé en termes de mode de perception du monde et, de ce fait, on quitte la logique de stigmatisation du manque pour entrer dans celle de la construction de la personne, qui passe par les pratiques d'observation, d'adaptation et de médiation.

Cette nouvelle logique repose essentiellement sur la mise à distance de l'idée, issue de l'approche médicale, que les difficultés rencontrées par un élève handicapé au cours de sa scolarité seraient à attribuer principalement à sa déficience ou à son trouble (sur le plan cognitif ou sensori-moteur), alors qu'elles peuvent le plus souvent être analysées en termes d'obstacles constitutifs d'une situation de handicap. Cette situation se définissant comme la résultante des interactions entre les caractéristiques singulières d'un élève et les contraintes de l'environnement scolaire dans lequel il est immergé. Pour créer les conditions favorables à la scolarisation de cet élève, il s'agira dès lors de réduire la situation de handicap par des démarches d'accompagnement appropriées ainsi que par la qualité de l'environnement matériel, physique et humain ${ }^{12}$. Dans le cas où ces médiations ne seraient pas mises en œuvre, C'est un Processus de production de handicap (PPH) qui se trouverait engagé (Fougeyrollas, 1999) et il est clair que la situation de handicap s'en trouverait aggravée, sans qu'on puisse l'imputer en aucune façon au trouble ou à la déficience.

12. Cf. circulaire 2002-111, du 30 avril 2002. 


\section{L'exemple de la situation d'Alain où l'échec de l'accompagnement social est producteur de handicap (source: enseignant référent - MDPH Vienne)}

Année 1: Alain est un enfant en surpoids scolarisé en CE2 qui manifeste un absentéisme chronique et un comportement agressif avec ses camarades. Sa mère, qui élève seule son enfant unique, a exprimée une forte demande d'aide éducative.

Année 2 et 3: Une mesure administrative d'Aide éducative en milieu ouvert (AEMO) intervient l'année suivante, mais elle n'a pas d'effet sur l'absentéisme, qui reste important, la mère n'étant pas "en mesure d'imposer l'école à son fils ". Une démarche est suggérée en direction de la MDPH en vue d'un " parcours de formation au sein d'un Itep ». La mère est accompagnée par les "partenaires sociaux » dans la constitution d'un dossier auprès de la MDPH de la Vienne. Ce dossier aboutit à une inscription dans un Itep situé en dehors du département.

Année 4: La scolarisation en Itep est marquée par un absentéisme très important. Alain, qui se plaint de mauvais traitements de la part de ses camarades, ne repart pas de chez sa mère après le week-end, celle-ci n'étant " toujours pas en mesure $d^{\prime}$ inciter Alain » à fréquenter une école.

Année 5: La mère a décidé "de retirer son fils de l'ltep " avec lequel elle n'entretient pas de bonnes relations. La décision MDPH est en fin de validité. Inscription au collège de secteur qu'il ne fréquente qu'une journée et demie par semaine. Constitution d'un dossier de renouvellement.

D'un point de vue systémique, le problème initial peut être analysé comme une double rupture des relations enfant école et parent école, liée à une situation familiale fragile. La demande familiale est de l'ordre de la reconstruction du lien social: la réponse par une mesure d'AEMO paraît adéquate. Mais celle-ci va se détourner de son objet en accompagnant la constitution d'un dossier MDPH, entraînant l'orientation d'Alain dans un établissement spécialisé lointain, dans lequel les difficultés initiales se trouveront reproduites, voire accentuées. On peut ici faire l'hypothèse que l'échec de la tentative de reconstruction du lien social en situation a entraîné une médicalisation du problème, désormais attribué à Alain, ce qui justifiait son orientation dans un établissement thérapeutique (Itep). Au bout du compte, quatre années ont passé, sans aucune évolution.

En revanche, l'identification, en contexte d'apprentissage, des besoins éducatifs particuliers des élèves permet d'échapper aux risques de stigmatisation et d'enfermement dans une catégorisation nosographique, elle préserve des effets pervers du déterminisme biologique ou psychopathologique.

\section{L'exemple de la situation de Tina, où le diagnostic de TED ${ }^{13}$ est mis à l'épreuve de la scolarisation (source: enseignant référent - MDPHVienne)}

Année 0:Tina, 3 ans, a été diagnostiquée TED et sa mère sans emploi, vivant seule avec sa fille, souhaite obtenir de la MDPH une prise en charge par un service médico-social. Elle dispose aussi d'un certificat médical préconisant un AVS. L'enseignant référent accompagne la mère dans la constitution d'un dossier MDPH. "L'observation à l'école de l'enfant " par les enseignants " ne va pas dans le sens de l'attribution d'une AVS ".

13. Trouble envahissant du développement. 
Année 1: Tina est scolarisée à temps partiel à la demande de la famille. À l'issue de l'équipe éducative organisée à l'école, des doutes sont émis quant au diagnostic d'autisme. En équipe pluridisciplinaire, la demande de PPCH fait l'objet d'un rejet. Appel de la famille qui obtient de la CDAPH le suivi par un service médico-social et une $\mathrm{AEEH}$ avec complément pour une année.

Année 2: À l'issue des ESS qui ont été organisées en liaison avec le service de soins, les observations conduisent à réduire progressivement les aides apportées. Lors de la seconde ESS, la mère convient qu'il paraît inutile de renouveler la demande de service médico-social. Sortie de Tina du champ du handicap.

On remarquera dans cette situation le caractère déterminant joué par l'observation en situation scolaire et pédagogique du fonctionnement réel de l'enfant. C'est en fait l'évaluation des besoins de Tina par les acteurs concernés de la scolarisation qui conduit à revoir le diagnostic et par conséquent les modalités de prise en charge. La perspective traditionnelle qui, dans le cadre d'un référentiel bio-médical, conduit à identifier les répercussions d'un trouble diagnostiqué et à y remédier se trouve ici renversée au profit d'une logique d'évaluation des besoins.

\section{Trouble ou besoin?}

Le troisième couple de notions correspond, une fois encore, à deux référentiels de pensée distincts : celui du trouble et celui du besoin. Le premier consiste à fonder la démarche pédagogique et l'action éducative sur la connaissance du trouble; le second, au contraire, consiste à partir de l'identification des obstacles rencontrés par l'élève dans la situation de vie et d'apprentissage pour déterminer ses besoins éducatifs particuliers: un élève rencontre un obstacle dans ses apprentissages ou dans sa vie familiale ou sociale, il en résulte un besoin d'aide qui est précisément constitutif du besoin éducatif particulier. Ce besoin éducatif n'est donc pas constitué préalablement à la situation d'enseignement, il est le produit des interactions qui la caractérisent. II serait certainement erroné de considérer ces deux modèles comme inconciliables, mais il n'en reste pas moins que les pratiques éducatives s'orienteront dans des directions très sensiblement différentes, selon que ces deux approches seront plus ou moins dominantes.

Parmi les principes de base de la formation aux diplômes du Capa-SH et 2CA-SH, il est mentionné dans le référentiel des compétences donné par la circulaire du 10 février 2004 que " ce n'est pas en soi le diagnostic qui détermine le choix de l'aide mais la nature des besoins de l'élève " (Annexe 1, I). II y a là, de manière évidente, une mise à distance de la recherche de l'étiologie du trouble, c'est-à-dire de la cause médicale des difficultés qui peuvent être observées. Et c'est en tant qu'elle serait déterminante pour l'action pédagogique et éducative que l'étiologie est mise à distance. Cette démarche permet d'une certaine façon d'échapper aux doctrines et de mettre en avant, d'une manière pragmatique, l'observation de l'enfant en situation d'apprentissage par l'enseignant de la classe et non pas seulement par l'enseignant référent. On n'est plus dans une logique de savoir dogmatique où tel type d'atteinte entraînerait inévitablement tels types de répercussions; on adopte une méthode dans laquelle on commence par observer, pour ensuite réfléchir aux modalités d'aide. Modalités d'aide qui consistent en fait à identifier les obstacles auxquels l'élève se heurte dans son environnement scolaire. On peut dire que tel 
élève a des difficultés pour comprendre les consignes, mais on peut aussi exprimer les choses différemment: on peut dire que cet élève ne comprendra la consigne $q u$ à la condition qu'on la lui explique de manière claire et qu'on la lui répète un certain nombre de fois. On peut dire que tel élève à des difficultés de repérage dans l'espace, mais on peut dire aussi que cet élève a besoin d'un cadre structuré pour se repérer dans l'espace. Ce n'est pas du tout la même approche. Le fait que les modalités de l'aide et les adaptations pédagogiques ne relèvent pas d'une prescription médicale, mais de l'observation de l'enseignant constitue un élément fort de son identité professionnelle dans le nouveau contexte inclusif de scolarisation des élèves en situation de handicap. La prégnance de l'approche médicale pourrait conduire à majorer le poids des pratiques de diagnostic a priori, fondées sur l'identification de besoins éducatifs a priori, construits comme l'envers positif de la répercussion du trouble, au regard de celles qui se fondent sur "l'inventivité régulée " (Meirieu, 1996). Les premières incluent le remède aux difficultés attribuées à l'élève dans les gestes professionnels et dans modes d'apprentissage proposés (Benayed et Verreman, 2011), tandis que les secondes consistent pour l'enseignant à ajuster au fur et à mesure les tâches d'apprentissage à partir de l'observation de leur déroulement effectif.

Mais s'il est vrai que le pédagogique ne dépend pas du médical, mais il peut être très fructueux que les personnels des services médico-sociaux réalisent leurs observations en situation scolaire. C'est alors que les données recueillies et mises en commun par les uns et par les autres pourront s'articuler de façon pertinente en vue de l'élaboration du PPS.

\section{CONCLUSION}

Dans un deuxième temps, un certain nombre d'informations spécifiques peuvent utilement être apportées, dont l'appropriation permettra l'ajustement des réponses adaptatives et des médiations spécifiques. L'éventail des possibilités de médiations pourrait être largement développé sous ses différents aspects: la prégnance du visuel, la nécessité d'un cadre structuré, le fait que le groupe ne soit pas un point de départ mais un objectif recherché... On voit bien que dans cette confrontation des deux référentiels de pensée fondés, l'un, sur la connaissance et l'étiologie du trouble, l'autre, sur les besoins éducatifs particuliers, la solution réside sans doute dans la recherche d'une articulation et d'un équilibre, dont la réalisation dépendra nécessairement de la mise en œuvre complémentaire des actions relevant de l'un et de l'autre. Si l'on admet que l'accompagnement des apprentissages d'un élève en situation de handicap s'accomplit tout au long d'un itinéraire pédagogique, on pourrait considérer, pour prolonger l'image, que l'enseignant qui remplit ce rôle a besoin de deux types de cartes pour s'orienter et pour orienter son travail. La première fonctionne comme une carte d'état-major, qui permet de connaître la nature de l'environnement immédiat et par conséquent de choisir le chemin le plus praticable; la seconde, comme une carte générale du relief, qui indique les grands obstacles à la progression, mer intérieure, lac, chaîne de montagnes, fleuve. Ainsi la politique de scolarisation des élèves en situation de handicap dans laquelle la loi nous engage aujourd'hui s'appuie-t-elle en premier lieu sur l'observation et l'analyse 
des besoins éducatifs particuliers, c'est-à-dire des obstacles qui peuvent survenir dans le milieu scolaire ordinaire, sans exclure de recourir ensuite, si nécessaire, à la lumière d'un certain nombre d'éléments d'ordre médical et psychologique, dont l'apport est de contribuer à la mise en accessibilité de la scolarité.

\section{Références}

Armstrong (F.), ARMstrong (D.), BARTON (L), eds, Inclusive Education. Policy, Contexts and Comparative Perspectives, David Fulton Publisher, London, 2000.

BARRAL (C.), " De I'influence des processus de normalisation internationaux sur les systèmes de pensée du handicap ", Handicap, n 81, janvier-mars 1999, CTNERHI, 1999.

BENAYED (M.), VERREMAN (A.), « ENT, apprentissage collaboratif et différenciation pédagogique: une possible symbiose? ", La nouvelle revue de l'adaptation et de la scolarisation, 55, p. 123-132.

BENOIT (H.), " De la déficience à l'obstacle », Cahiers pédagogiques, 459, in dossier "L'école à l'épreuve du handicap », Crap, 2008a, p. 51.

BENOIT (H.), « De la reproduction des pratiques à leur transformation: le défi de la formation des enseignants ", Reliance, 27, dossier "Faire culture commune ", Érès, 2008b, p. 99-104.

BENOIT (H.), "Du détour ségrégatif à la scolarisation des élèves handicapés ", Éducation \& Management, CRDP de l'académie de Créteil, septembre 2006.

BENOIT (H.), "Handicap et intégration: du détour ségrégatif à l'école inclusive ", La nouvelle revue de I'AIS, 28, dossier " 1954-2004: 50 ans de formations spécialisées ", Éditions du Cnefei, Suresnes, 2004, p. 27-33.

BENOIT (H.), "Peut-on parler de besoins éducatifs particuliers en cas de difficulté scolaire? ", La nouvelle revue de I'AIS, 22, 2003, p. 81-88.

CANAT (S.), "L'inclusion: I'accueil de la différence ", La nouvelle revue de I'adaptation et de la scolarisation, hors série n 5, INS HEA, 2009, p. 147-156.

CHAMPOLLION (P.), "Approche comparative des systèmes éducatifs européens de scolarisation des jeunes handicapés ", La nouvelle revue de l'adaptation et de la scolarisation, 37, 2007, p. 215-223.

ChAUVIÈRE (M), PLAISANCE (É.), "Les conditions d'une culture partagée ", Reliance, 27, dossier "Faire culture commune », Érès, 2008, p. 31-44.

CHAUVIÈRE (M), PLAISANCE (É.), L'école face aux handicaps. Éducation spéciale ou éducation intégrative?, PUF, Paris, 2000.

D'ALESSIO (S.), " 30 ans d'Integrazione scolastica en Italie ", La nouvelle revue de l'adaptation et de la scolarisation, hors série n 5, INS HEA, 2009, p. 35-50.

GARDOU (C.), JEANNE (Y.), dir., "Faire culture commune » dossier, Reliance, 27, Érès, 2008, p. 29-120.

FOUGEYROLLAS (P.), " Entretien avec Fougeyrollas. Propos recueillis par Mouloud Boukala ", La nouvelle revue de l'adaptation et de la scolarisation, 45, p. 165-174. 
FOUGEYROLLAS (P.), « RIPPH. Le réseau international sur le processus de production du handicap (RIPPH) : mieux définir pour mieux comprendre, mieux comprendre pour mieux intervenir ". Handicap, revue de sciences humaines et sociales, 1999, 83, p. 115-118.

GENEVOIS (G.), « Incidence du déficit visuel sur le développement psychologique et prise en compte de la différence ", La nouvelle revue de I'AIS, 22, 2003, p. 127-137. HORVAIS (J.), "Une (nouvelle) mission qui questionne l'école. Regards d'un institut médico-éducatif ", La nouvelle revue de l'adaptation et de la scolarisation, 47, 2009, p. 195-206.

MEIRIEU (P.), "La pédagogie différenciée: enfermement ou ouverture » in Alain BENTOLILA, L'école: diversités et cohérence, Nathan, Paris, 1996, p. 109-149.

STICKER, (H.-J.), Corps infirmes et société, nouv. éd., Dunod, Paris, 1997.

VALLERIE (B.), "Aider au développement du pouvoir d'agir des personnes en situation de handicap ", La nouvelle revue de l'adaptation et de la scolarisation, 51, 2010, p. 271-282. 